\title{
Preparation of films of a highly aligned lipid cubic phase
}

Article

Published Version

Squires, A., Hallett, J. E., Beddoes, C. M., Plivelic, T. S. and Seddon, A. M. (2013) Preparation of films of a highly aligned lipid cubic phase. Langmuir, 29 (6). pp. 1726-1731. ISSN 0743-7463 doi: https://doi.org/10.1021/la304726m Available at https://centaur.reading.ac.uk/31172/

It is advisable to refer to the publisher's version if you intend to cite from the work. See Guidance on citing.

To link to this article DOI: http://dx.doi.org/10.1021/la304726m

Publisher: American Chemical Society

All outputs in CentAUR are protected by Intellectual Property Rights law, including copyright law. Copyright and IPR is retained by the creators or other copyright holders. Terms and conditions for use of this material are defined in the End User Agreement.

\section{www.reading.ac.uk/centaur}

\section{CentAUR}

Central Archive at the University of Reading

Reading's research outputs online 


\title{
Preparation of Films of a Highly Aligned Lipid Cubic Phase
}

\author{
Adam M. Squires, ${ }^{* \dagger \dagger}$ James E. Hallett, ${ }^{\ddagger}, \|, \S$ Charlotte M. Beddoes, ${ }^{\ddagger, \|}$ Tomás S. Plivelic, ${ }^{\perp}$ \\ and Annela M. Seddon $*,+, \S$ \\ ${ }^{\dagger}$ Department of Chemistry, Whiteknights Campus, University of Reading, Reading RG6 6AD, United Kingdom \\ ${ }^{\ddagger}$ Bristol Centre for Functional Nanomaterials, Nanoscience and Quantum Information Building and ${ }^{\S}$ H. H. Wills Physics Laboratory, \\ Tyndall Avenue, University of Bristol, Bristol BS8 1FD, United Kingdom \\ "School of Chemistry, Cantock's Close, University of Bristol, Bristol BS8 1TD, United Kingdom \\ ${ }^{\perp}$ MAX IV Laboratory, Lund University, SE-221 00 Lund, Sweden
}

\section{Supporting Information}

ABSTRACT: We demonstrate a method by which we can produce an oriented film of an inverse bicontinuous cubic phase $\left(\mathrm{Q}_{\mathrm{II}}{ }^{\mathrm{D}}\right)$ formed by the lipid monoolein (MO). By starting with the lipid as a disordered precursor (the $\mathrm{L}_{3}$ phase) in the presence of butanediol, we can obtain a film of the $\mathrm{Q}_{\mathrm{II}}{ }^{\mathrm{D}}$ phase showing a high degree of in-plane orientation by controlled dilution of the sample under shear within a linear flow cell. We demonstrate that the direction of orientation of the film is different from that found in the oriented bulk material that we have reported previously; therefore, we can now reproducibly

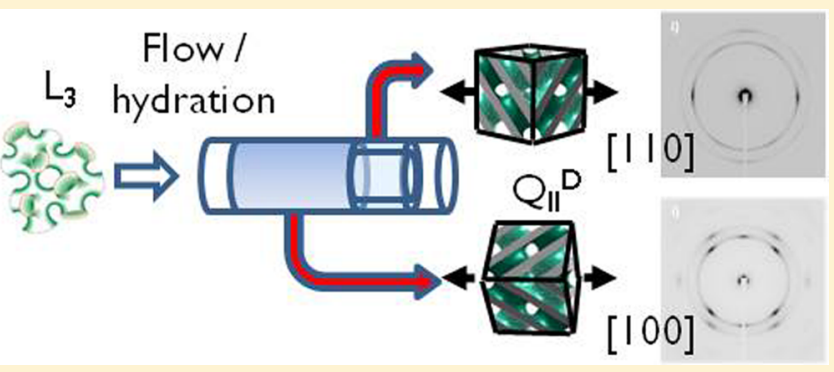
form $\mathrm{Q}_{\mathrm{II}}^{\mathrm{D}}$ samples oriented with either the $[110]$ or the [100] axis aligned in the flow direction depending on the method of preparation. The deposition of MO as a film, via a moving fluidair interface that leaves a coating of $\mathrm{MO}$ in the $\mathrm{L}_{3}$ phase on the capillary wall, leads to a sample in the [110] orientation. This contrasts with the bulk material that we have previously demonstrated to be oriented in the [100] direction, arising from flow producing an oriented bulk slug of material within the capillary tube. The bulk sample contains significant amounts of residual butanediol, which can be estimated from the lattice parameter of the $\mathrm{Q}_{\mathrm{II}}{ }^{\mathrm{D}}$ phase obtained. The sample orientation and lattice parameters are determined from synchrotron small-angle X-ray scattering patterns and confirmed by simulations. This has potential applications in the production of template materials and the growth of protein crystals for crystallography as well as deepening our understanding of the mechanisms underlying the behavior of lyotropic liquid-crystal phases.

\section{INTRODUCTION}

We have previously demonstrated a method to produce highly oriented inverse bicontinuous cubic phases of biological lipids. ${ }^{1}$ In this Letter, we show that we are able to produce oriented films of this material by a modification of this method, which displays a different crystallographic orientation.

The inverse bicontinuous cubic phases formed by biological amphiphiles, such as lipids, are of great interest across of a range of scientific disciplines. ${ }^{2}$ These $3 \mathrm{D}$ nanostructures are composed of a curved fluid bilayer made from the lipid molecules, which separates two interpenetrating continuous networks of water channels. Three such cubic phases are known: the $\mathrm{Q}_{\mathrm{II}}^{\mathrm{D}}$ (diamond), $\mathrm{Q}_{\mathrm{II}}{ }^{\mathrm{P}}$ (primitive), and $\mathrm{Q}_{\mathrm{II}}^{\mathrm{G}}$ (gyroid) phases, each of which contains water channels typically $2-5 \mathrm{~nm}$ in diameter. Because of their high surface area and easily tuned geometry, they have potential as templates for size- selective molecular sieves or catalysts ${ }^{34}$ or for electronic applications. ${ }^{56}$ They have also shown success as a matrix for the crystallization of membrane proteins ${ }^{7}$ and as a host for siRNA ${ }^{8}$. Moreover, given the biological origin of the amphiphiles and the similarity of the bilayer environment within the $Q_{I I}$ phase to a cell membrane, they have much more widespread potential in protein research. ${ }^{10}$ A related but much less ordered phase than the inverse bicontinuous cubics has also been reported: the $\mathrm{L}_{3}$ or sponge phase. The $\mathrm{L}_{3}$ phase can be considered to be a disordered cubic phase that still displays a bicontinuous network of water channels separated by a lipid bilayer. ${ }^{11}$ It possesses short-range order; however, it is disordered over longer length scales. Amphiphiles that spontaneously form cubic phases are often seen to form $\mathrm{L}_{3}$ phases in the presence of additives, which causes a relaxation of the curvature of the cubic phase, leading to this disordered structure. ${ }^{12,13}$

We recently reported a method by which the $L_{3}$ phase formed by biological lipids could be converted to a highly oriented $\mathrm{Q}_{\mathrm{II}}^{\mathrm{D}}$ phase using controlled dilution under shear. ${ }^{1} \mathrm{An}$ oriented sample such as this is advantageous because it allows information to be obtained that is not possible from the unoriented analogue. This includes the mechanism of interconversion of lyotropic phases, which cannot be determined unambiguously using powderlike samples. ${ }^{14}$ In

Received: May 24, 2012

Revised: January 18, 2013

Published: January 24, 2013 
addition, internal domain boundaries are also known to affect phase-transition kinetics, ${ }^{15}$ and can potentially affect transport, mechanical, and optical properties ${ }^{16}$ in cubic phases and inorganic materials templated from them. In such applications, it is likely that key properties are dependent on the control of the specific orientation adopted. In a different approach, Pieranski has produced single crystallites of an inverse bicontinuous cubic phase between $10 \mu \mathrm{m}$ and $1 \mathrm{~mm}$ in size. ${ }^{17}$ although no direct evidence for the orientation has been produced using X-ray scattering, the observed faceting of the droplet makes it highly likely that the sample is macroscopically oriented.

Although much work has concentrated on the production of bulk materials of lipid cubic phases, there has been little previous work on the formation of inverse bicontinuous cubic phases of lipids as films. This has previously been achieved by dip coating the lipid from a chloroform solution, followed by hydratio, for subsequent visualization with atomic force microscopy. ${ }^{18}$ However, this method does not produce a material with in-plane orientation, and the production and analysis of such films are nontrivial. By preparing a film from the $\mathrm{L} 3$ phase, however, the relatively low viscosity of the $\mathrm{L}_{3}$ precursor $^{19}$ can be exploited. Furthermore, as we will demonstrate here, it is possible to prepare a film with inplane orientation.

The bulk $\mathrm{Q}_{\mathrm{II}}^{\mathrm{D}}$ phases produced in previous work were found to be oriented with the [100] axis aligned parallel to the flow direction. We show here that it possible to produce an oriented thin film of the same material and that this film displays a different orientation, with the [110] axis aligned parallel to the flow direction. This is achieved by setting the flow conditions such that the film is formed on the X-ray capillary as the liquid-air interface passes repeatedly across the portion of the sample that is being measured by the X-ray beam. By achieving control over the deposition of the material and the subsequent structural alignment, it opens up further possibilities for the use of materials in applications where a particular crystal plane may be desirable or where a thin film may be advantageous.

\section{MATERIALS AND METHODS}

Experimental Data. A 60:40 v/v solution of water/1,4-butanediol was prepared and mixed with monoolein (MO) (Rylo, received as a gift from Danisco) in a 60:40 w/w solvent/MO ratio. The sample underwent two freeze-thaw cycles to form an optically isotropic viscous liquid. Samples were loaded into a custom-built syringe-pumpdriven flow cell using 1.5-mm-internal-diameter fluorinated ethylene propylene (FEP) tubing connected to a $1.5-\mathrm{mm}$-diameter thin-walled borosilicate glass X-ray capillary.

The method of preparation of a thin film oriented in the [110] direction is illustrated in Figure 1. First, approximately $50 \mu \mathrm{L}$ of the $\mathrm{L}_{3}$ phase was introduced into the capillary at a distance such that when oscillatory shear was applied the air $-\mathrm{L}_{3}$ interface passed through the X-ray beam. SAXS patterns confirmed that the starting material was present in the $\mathrm{L}_{3}$ phase. The sample was separated from a column of water by an air gap of approximately $40 \mu \mathrm{L}$. The sample was subjected to oscillatory shear at a rate of $10 \mu \mathrm{L} \mathrm{s}^{-1}$ with a displacement of $50 \mu \mathrm{L}$ (Figure 1i-iv). The acquisition of SAXS data during this process demonstrated the formation of an oriented cubic phase, which remained stable when the capillary was subsequently flushed with water (step v). As a mechanism for cubic-phase formation, we suggest that the water and sponge phases each leave a residual coating in their path on every passage, and because the $50 \mu \mathrm{L}$ displacement is greater than the $40 \mu \mathrm{L}$ air gap, there is some overlap, allowing the gradual exchange of material. This dilution reduces the concentration of butanediol, causing cubic-phase formation, as we have discussed

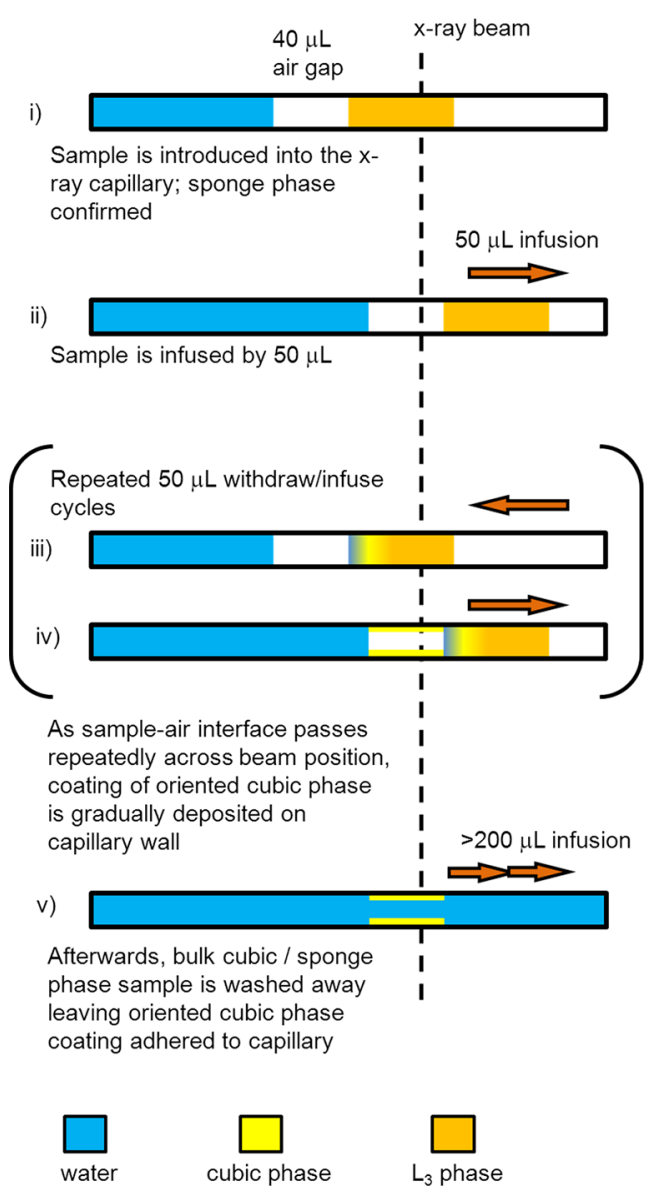

Figure 1. Schematic showing the process by which the oriented cubic phase film is formed within the linear flow cell. Under steps $\mathrm{i}-\mathrm{iv}$, a sample-air interface repeatedly crosses the $\mathrm{X}$-ray beam path, leaving a thin film of sample adhering to the capillary wall. When the bulk cubic/sponge phase is washed away (step v), the remaining cubic phase coating is found to be oriented with the [110] axis parallel to the flow direction.

previously. ${ }^{1}$ Our previous studies have demonstrated that the bulk slug of sample to the right itself gradually becomes transformed into the cubic phase showing a [100] orientation. ${ }^{1}$

Data were collected at beamline I911-4 at the Max-lab synchrotron facility ${ }^{20}$ with a beam size at the sample of $0.3 \times 0.3 \mathrm{~mm}^{2}$ (full width at half-maximum, fwhm). The wavelength was $0.91 \AA$, and data were collected over a $q$ range of $0.006-0.18 \AA^{-1}$. Images were recorded in a bidimensional CCD detector ( $165 \mathrm{~mm}$ diameter from Marresearch Inc.) over a range of exposure times between 30 and $60 \mathrm{~s}$, and azimuthal and radial plots were generated using the YAX macro within Image.$^{21}$ Additional data in the linear flow cell were collected at beamline I22 at the Diamond Light Source and recorded on a Pilatus 2 $\mathrm{M}$ detector. Data in a Couette cell were collected at the ID02 highbrilliance beamline at the ESRF using a Haake RS300 rheometer fitted with an X-ray-transparent polyimide Couette cell (inner diameter, 20 $\mathrm{mm}$; outer diameter, $22 \mathrm{~mm}$; height, $40 \mathrm{~mm}$; gap, $1 \mathrm{~mm}$ ). For the azimuthal plots shown in Figures $2 b-d$ and $3 b, c$, a background was subtracted as determined by the average of azimuthal plots from annuli taken inside and outside each ring. ${ }^{22}$ For Figure $3 d$, this could not be done because of the small signal to noise ratio, and thus a constant background was subtracted.

Simulated Data. Simulated peak positions were calculated as follows; we denote the uniaxial axis of rotation, which lies parallel to the flow direction, as $\left[h^{\mathrm{R}} k^{\mathrm{R}} l^{\mathrm{R}}\right]$. (This axis is [100] or [110] in the two different orientations described in this Letter.) For a given $(h k l)$ reflection, we begin by identifying every possible $\{h k l\}$ combination. For example, for $\{h k l\}=\left\{\begin{array}{lll}1 & 0 & 0\end{array}\right\}$, the six variations of $(h k l)$ are $\left(\begin{array}{lll}1 & 0 & 0\end{array}\right)$, 


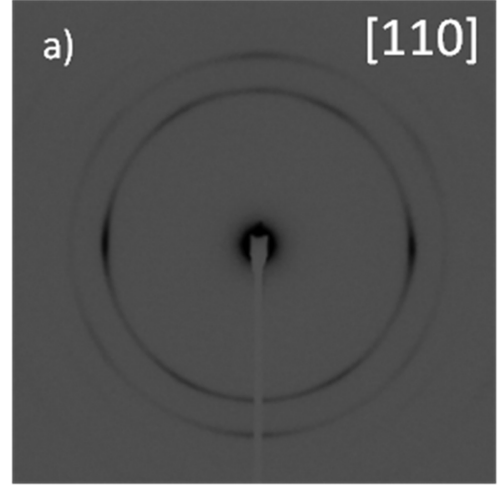

c)

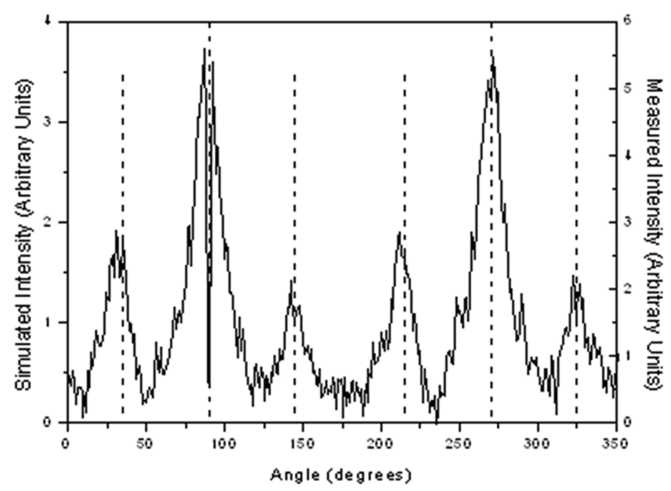

b)

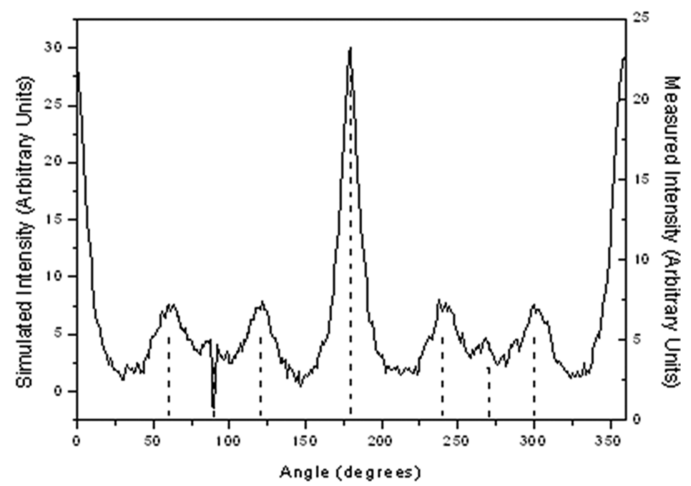

d)

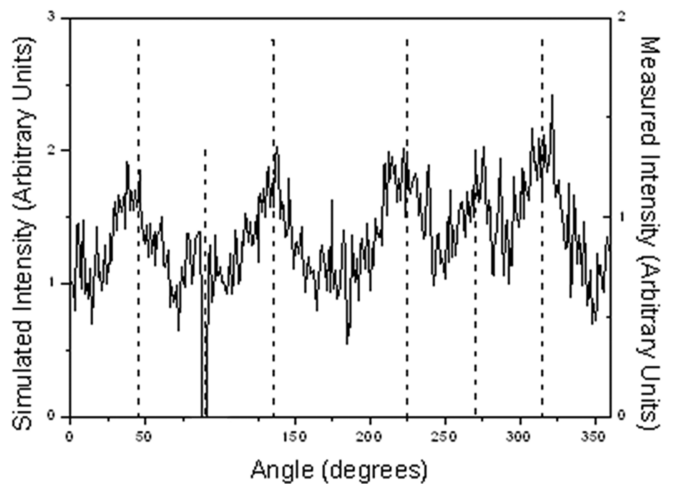

Figure 2. (a) 2D SAXS pattern for a sample oriented along the [110] axis. (b-d) Aziumuthally integrated 1D patterns of a sample oriented along the [110] axis, with (b) the first reflection, (c) the second reflection, and (d) the third reflection. The dotted lines indicate the positions of the expected peaks in the azimuthally integrated data based on our simulations. Angles are relative to the flow direction, which is horizontal in the 2D SAXS patterns.

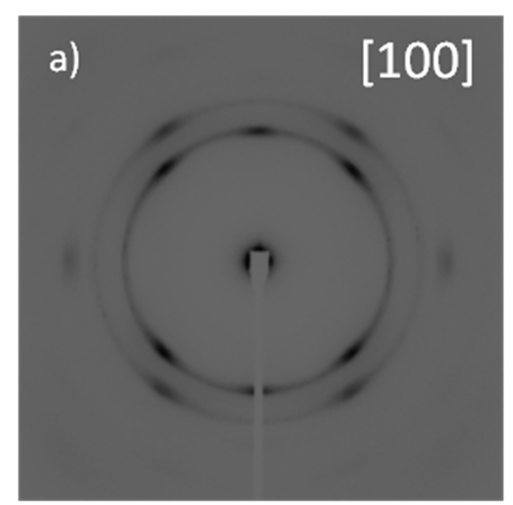

c)

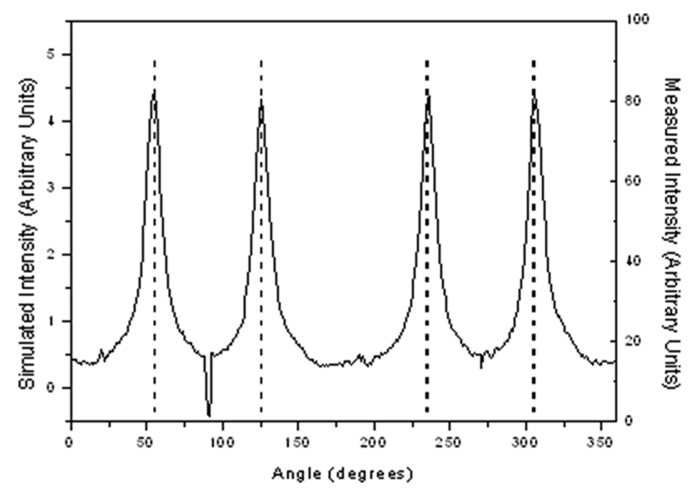

b)

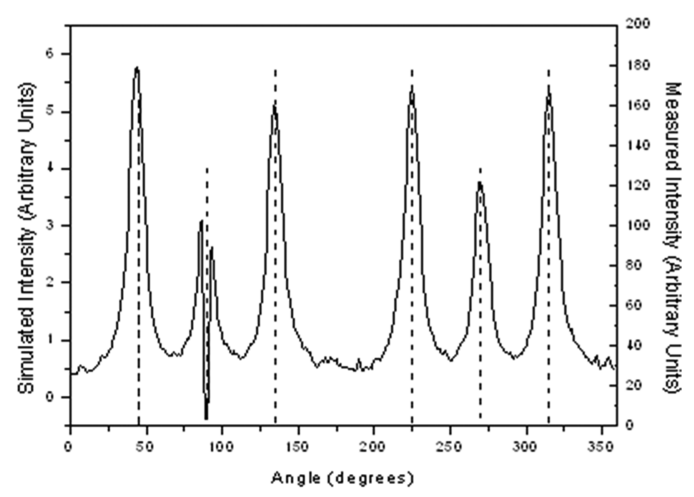

d)

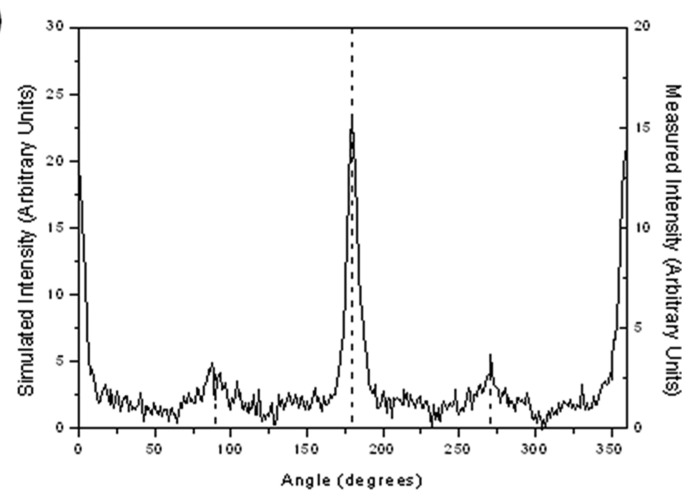

Figure 3. (a) 2D SAXS pattern for a sample oriented along the [100] axis. (b-d) Aziumuthally integrated 1D patterns of a sample oriented along the [100] axis, with (b) the first reflection, (c) the second reflection, and (d) the third reflection. The dotted lines indicate the positions of the expected peaks in the azimuthally integrated data based on our simulations. Angles are relative to the flow direction, which is horizontal in the 2D SAXS patterns. 
$\left(\begin{array}{lll}-1 & 0 & 0\end{array}\right),\left(\begin{array}{lll}0 & 1 & 0\end{array}\right),\left(\begin{array}{lll}0 & -1 & 0\end{array}\right),\left(\begin{array}{lll}0 & 0 & 1\end{array}\right)$, and $\left(\begin{array}{lll}0 & 0 & -1\end{array}\right)$. Each $\{h k l\}$ variation gives two reflections with azimuthal angles of $\pm \chi$ relative to the axis of rotation, which is horizontal in the images shown. The azimuthal angle, $\chi$, is approximately equal to the angle between the $\left[h^{\mathrm{R}} k^{\mathrm{R}} l^{\mathrm{R}}\right]$ direction and the $[h k l]$ vector that is normal to the $(h k l)$ planes and can be described as

$$
\cos (\chi)=\frac{\left[h^{\mathrm{R}} k^{\mathrm{R}} l^{\mathrm{R}}\right] \cdot[h k l]}{\left|\left[h^{\mathrm{R}} k^{\mathrm{R}} l^{\mathrm{R}}\right] \|[h k l]\right|}
$$

where $\left[\left[h^{\mathrm{R}} k^{\mathrm{R}} l^{\mathrm{R}}\right] \mid\right.$ is the modulus of vector $\left[h^{\mathrm{R}} k^{\mathrm{R}} l^{\mathrm{R}}\right]$.

This approximation is valid for scattering at small angles where reflections occur when the $[h k l]$ direction lies approximately in the plane of the detector and therefore perpendicular to the beam direction, which is close to parallel to the $(h k l)$ planes. Because the $\mathrm{X}$ ray wavelength is $0.91 \AA$ and the $d$ spacings of the reflections were $>50$ $\AA$, this approximation is reasonable. We also determined a qualitative estimate of the relative intensities of reflections in a given azimuthal scan for different sets of $\{h k l\}$ planes. Our estimated relative intensity is given by the multiplicity divided by $\sin (\chi)$ and reflects the Lorentz factor for uniaxial (i.e., fiber-averaged) scattering patterns. ${ }^{23}$ The multiplicity in this case is the number of $\langle h k l\rangle$ vectors giving reflections at the same values of $\chi$. We have arbitratily assigned an upper limit of 30 for the relative intensity when $\chi=0$ in order that the value remain finite. Because of the various approximations made in our theoretical approach and the manner in which the background subtraction was performed on the experimental data, this estimate is not quantitative; it predicts which reflections are more intense than others but does not give the exact ratio of intensities.

\section{RESULTS AND DISCUSSION}

Figure 2 shows the 2D scattering pattern obtained from the oriented film and its corresponding integrated 1D azimuthal plots for the first three reflections. The data clearly demonstrate a significant degree of alignment. Because the data were obtained with the beam passing perpendicular to the plane of the substrate (i.e., across the diameter of the capillary tube), this alignment is parallel to the plane of the substrate. We demonstrate that the data are consistent with a sample uniaxially oriented about the [110] axis that lies parallel to the flow direction by including simulations for the predicted azimuthal angles of each reflection, assuming this orientation. The simulated positions and approximate relative intensities are shown as dotted lines, which give good agreement with the experimental data. The sample was left sealed in the capillary and was found to be stable in this orientation for over $24 \mathrm{~h}$ (Supporting Information, Figure S2). The possibility of a sample maintaining its orientation on these much longer time scales greatly enhances the potential value of these oriented materials.

Figure 3 shows the 2D scattering pattern and corresponding azimuthal plots for an oriented bulk sample prepared using the method described previously. ${ }^{1}$ Here, we find that the orientation matches the pattern generated by a sample oriented in the [100] direction.

We have considered a number of possible mechanisms that could explain the in-plane orientation of the film sample and the reasons that it produces an orientation that is different from that observed previously for the bulk sample.

First, there is the possibility that a thin coating of the sponge phase is rapidly converted to an unoriented cubic phase, which is then oriented by the shear force of the flowing water. The shear force that is experienced is greatest at the walls and decreases closer to the center of the capillary. We estimate that given a volume flow rate of $10 \mu \mathrm{L} \mathrm{s}^{-1}$ in a capillary of radius $0.75 \mathrm{~mm}$, the shear rate at the wall would be $30 \mathrm{~s}^{-1}$. Moreover, as the thin films experience a higher shear rate than the bulk sample closer to the center of the capillary and analogous work on gyroid diblock copolymers describes the selection of a particular orientation under increasing shear, ${ }^{24}$ this could in principle suggest the difference in orientation between the thinfilm samples described here and the oriented bulk samples in our previous paper. To test this, we formed a thin film of $\mathrm{MO}$ on the inside of the capillary. To provide this thin coating of lipid, a $50 \% \mathrm{w} / \mathrm{w}$ solution of $\mathrm{MO}$ in ethanol was introduced into the capillary and then removed, and the residual solvent was allowed to evaporate. This has been shown to leave a thin coating of lipid approximately $10 \mu \mathrm{m}$ thick. ${ }^{6}$ Water was then introduced under oscillatory shear in a manner analogous to that described in the previous experiments. We observed that films prepared from ethanol were hydrated immediately to give a $\mathrm{Q}_{\mathrm{II}}^{\mathrm{D}}$ phase. However, when this film was subjected to repeated oscillatory shear with water present, the sample did not display any orientation (Supporting Information, Figure S3). We also considered the possibility that the presence of some residual butanediol would soften the cubic phase, making it more suspectible to the shear orientation. To test this, we replaced the water with an aqueous solution of 15 wt \% butanediol. However, repeated shear with this solution did not orient the cubic phase, even with an increased flow rate of 30 $\mu \mathrm{L} \mathrm{s}^{1-}$ (Supporting Information, Figure S4i,ii).

Second, we considered the possibility that shear acting on a film of the sponge phase causes it to orient, prior to its transformation to a $\mathrm{Q}_{\mathrm{II}}^{\mathrm{D}}$ phase, as previous work on surfactant sponge phases has shown the transformation of the $\mathrm{L}_{3}$ phase to an aligned lamellar phase under shear. ${ }^{25}$ However, even subjecting the sponge phase to a shear rate of $500 \mathrm{~s}^{-1}$ in a Couette cell is insufficient to produce any orientation of the sponge alone (Supporting Information, Figure S5i,ii).

We therefore suggest that there is something inherent in the simultaneous hydration and shear that causes the orientation, both for the thin film and for the oriented bulk samples described previously. It is difficult to separate further the variables of shear rate, the rate of dilution of the butanediol, and the thin film versus bulk morphology of the sample. It is possible, for example, that the difference in orientation between the thin films and the bulk samples could be ascribed to the differences in residual butanediol concentration, as demonstrated by the differences in the lattice parameter shown later. It has been suggested that such a hypothesis could in principle be tested by carrying out the experiments with different concentrations of butanediol already dissolved in the column of water driven by the syringe pump. However, in practice we have not successfully obtained samples with any orientation using this approach, so we cannot test this hypothesis; it is possible that the presence of butanediol itself affects the surface tension at the liquid-air interface, in addition to changing the physical properties of the mesophase itself. Nonetheless, it is unclear how a change in butanediol concentration could cause a complete change from one orientation to another, and we hypothesize, given the current evidence, that a more plausible explanation is that the different orientation in the film is brought about by the passage of the liquid-air interface over the sample that is in the X-ray beam in a manner analogous to the "molecular combing" process that has been shown previously to cause alignment in samples made of protein fibrils. $^{26,27}$ This does not occur in the formation of the bulk samples reported previously, where orientation is induced by bulk flow. 
Radial integration (over the full $360^{\circ}$ range of azimuthal angles) of the $2 \mathrm{D}$ patterns shown above allows us to calculate and compare the lattice parameters for each sample. These are shown in Figure 4, plotted against time to emphasize the

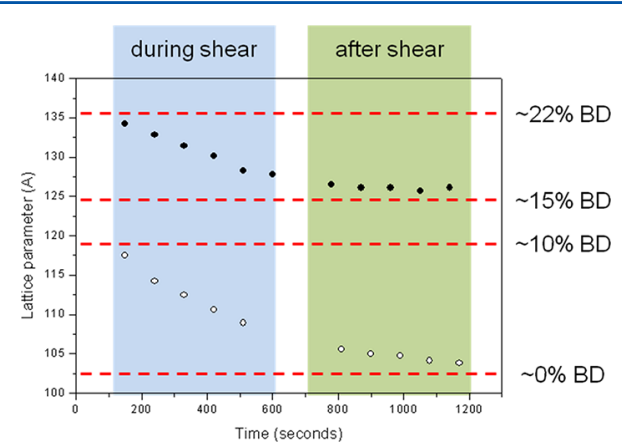

Figure 4. Measured lattice parameters for the cubic phase measured as a function of time. The [100] orientation is shown as filled circles; the [110] orientation is shown as open circles. Red lines indicate the estimated butanediol concentration present in the data shown in Cherezov et al. ${ }^{12}$

decrease in the lattice parameter in which dilution under shear occurs for both orientations. Note, however, that the times are approximate because no trigger mechanism was used during data acquisition. For the thin-film sample oriented in the [110] direction, there is a similar trend in the decrease in the lattice parameter with time under shear, followed by a stabilization of the lattice parameter after shear is halted. However, now the initial value is $117.5 \AA$, decreasing to $108.9 \AA$ under shear and finally reaching a value of $103.8 \AA$, which is within the error for the expected value for a $\mathrm{Q}_{\mathrm{II}}^{\mathrm{D}}$ phase formed by $\mathrm{MO}$ in excess water. $^{28}$ Although the initial lattice parameter and subsequent measurements of the [110]-oriented phase are swollen with respect to what would be expected if no butanediol were present, they are smaller than the values obtained for the [100]oriented sample. The final value measured for the [110]oriented sample is close to that for a $\mathrm{Q}_{\mathrm{II}}^{\mathrm{D}}$ phase in pure water. By comparing the observed lattice parameters in our oriented material with those observed by Cherezov et al, ${ }^{12}$ it can be seen that the [110] orientation contains less butanediol than the [100] orientation, and it is likely by the end of the experiment that it contains no butanediol. We find that for the bulk sample oriented in the [100] direction, the initial value of the lattice parameter is swollen relative to the lattice parameter expected for a $\mathrm{Q}_{\mathrm{II}}^{\mathrm{D}}$ phase formed by $\mathrm{MO}$ in excess water, which is approximately $104 \AA^{28}$ The initially observed value of $134.2 \AA$ gradually decreases to $127.8 \AA$ under continued oscillatory shear. Shear is then halted, and the lattice parameter undergoes a further decrease to $126.1 \AA$, at which point it appears to stabilize. A scattering pattern was measured approximately $3 \mathrm{~h}$ after shear was halted, and the sample was found to have retained some orientation, though it was not oriented to the same degree as immediately after preparation. After $3 \mathrm{~h}$, the lattice parameter was measured to be $125.5 \AA$ (data shown in the Supporting Information, Figure S1). This initial increase in the lattice parameter is to be expected; the presence of butanediol used to form the sponge phase leads to an increase in the lattice parameter, as observed by Cherezov et al. ${ }^{12}$ as well as us in our previous work. ${ }^{1}$

The orientation of the sample in the [110] direction was achieved when, as described above, there was repeated movement of the liquid-air interface across the X-ray beam, which lead to the "painting" of a thin film of the $\mathrm{L}_{3}$ phase on the capillary wall. The $d$ spacing of the resulting [110] orientation is comparable to the initial value described above (data not shown). In both instances, the common factors are the removal of greater amounts of butanediol from the sample (as shown by the decrease in $d$ spacing) and the potential to form a thin film on the walls of the capillary. It is possible that one of these is the prevailing mechanism or indeed that they may be dependent upon each other.

However, one advantage of our method is the relatively low viscosity of the $\mathrm{L}_{3}$ precursor ${ }^{19}$ from which the oriented $\mathrm{Q}_{\mathrm{II}}{ }^{\mathrm{D}}$ phase is formed. Thus, our ability to form thin films of oriented material, which can be introduced to an area of interest on a surface using a low-viscosity precursor, opens up a greater scope for the use of this method in the production of ordered nanoscale materials, for example, in templating applications.

\section{CONCLUSIONS}

We have demonstrated that it is possible to control the alignment of a uniaxially oriented $\mathrm{Q}_{\mathrm{II}}^{\mathrm{D}}$ phase with either the [110] or [100] axis aligned parallel to the flow direction. This has been achieved by controlled dilution under shear, and it appears to be a function of the total or partial removal of butanediol from the sample. In cases where the sample is present as a thin film, the removal of butanediol is complete and the orientation is along the [110] direction. In bulk samples, this removal is partial and leads to the formation of a swollen oriented $\mathrm{Q}_{\mathrm{II}}^{\mathrm{D}}$ phase as seen previously. The control of both the production of thin film versus bulk materials and the orientation in this way has potential in applications such as the production of templated materials, where the selection of a particular orientation may be beneficial. Furthermore, it may open up further insights into the mechanisms of interconversion between inverse bicontinuous cubic phases that require the sample to be oriented.

\section{ASSOCIATED CONTENT}

\section{S Supporting Information}

Two-dimensional SAXS images and integrated data showing sample stability with time. This material is available free of charge via the Internet at http://pubs.acs.org.

\section{AUTHOR INFORMATION}

\section{Corresponding Author}

*E-mail: a.m.squires@reading.ac.uk. Tel: +44 (0)118 3784736. E-mail: annela.seddon@bristol.ac.uk. Tel: +44 (0)117 394 0015;

\section{Notes}

The authors declare no competing financial interest.

\section{ACKNOWLEDGMENTS}

J.E.H. and C.B. are supported by EPSRC CDT grant EP/ G036780/1. We thank MAX-lab for the provision of beam time under proposal 20110300.

\section{REFERENCES}

(1) Seddon, A. M.; Lotze, G.; Plivelic, T. s. S.; Squires, A. M. A Highly Oriented Cubic Phase Formed by Lipids under Shear. J. Am. Chem. Soc. 2011, 133, 13860-.

(2) Kulkarni, C. V.; Wachter, W.; Iglesias-Salto, G.; Engelskirchen, S.; Ahualli, S. Monoolein: A Magic Lipid? Phys. Chem. Chem. Phys. 2011, 13, 3004-. 
(3) Negrini, R.; Mezzenga, R. Diffusion, Molecular Separation, and Drug Delivery from Lipid Mesophases with Tunable Water Channels. Langmuir 2012, 28, 16455-.

(4) Holyst, R. Infinite Networks of Surfaces. Nat. Mater. 2005, 4, 584-.

(5) Wang, D. H.; Luo, H. M.; Kou, R.; Gil, M. P.; Xiao, S. G.; Golub, V. O.; Yang, Z. Z.; Brinker, C. J.; Lu, Y. F. A General Route to Macroscopic Hierarchical 3D Nanowire Networks. Angew. Chem., Int. Ed. 2004, 43, 6169-.

(6) Akbar, S.; Elliott, J. M.; Rittman, M.; Squires, A. M. Adv. Mater. 2012, DOI: $10.1002 /$ adma.201203395.

(7) Caffrey, M. Crystallizing Membrane Proteins for Structure Determination: Use of Lipidic Mesophases. Ann. Rev. Biophys. 2009, 38, 29.

(8) Leal, C. 1.; Bouxsein, N. F.; Ewert, K. K.; Safinya, C. R. Highly Efficient Gene Silencing Activity of siRNA Embedded in a Nanostructured Gyroid Cubic Lipid Matrix. J. Am. Chem. Soc. 2010, 132, 16841-.

(9) Mulet, X.; Boyd, B. J.; Drummond, C. J. Advances in Drug Delivery and Medical Imaging Using Colloidal Nanoparticulate Lyotropic Liquid Crystalline Dispersions. J. Colloid Interface Sci.2012, doi: 10.1016/j.jcis.2012.10.014.

(10) Bilewicz, R.; Rowinski, P.; Rogalska, E. Modified Electrodes Based on Lipidic Cubic Phases. Bioelectrochemistry 2005, 66, 3.

(11) Porte, G. Isotropic Phases of Bilayers. Curr. Opin. Colloid Interface Sci. 1996, 1, 345-.

(12) Cherezov, V.; Clogston, J.; Papiz, M. Z.; Caffrey, M., Room to Move: Crystallizing Membrane Proteins in Swollen Lipidic Mesophases. J. Mol. Biol. 2006, 357, 1605.

(13) Wadsten-Hindrichsen, P.; Bender, J.; Unga, J.; Engstrom, S. Aqueous Self-Assembly of Phytantriol in Ternary Systems: Effect of Monoolein, Distearoylphosphatidylglycerol and Three Water-Miscible Solvents. J. Colloid Interface Sci. 2007, 315, 701-.

(14) Squires, A. M.; Templer, R. H.; Seddon, J. M.; Woenkhaus, J.; Winter, R.; Narayanan, T.; Finet, S. Kinetics and Mechanism of the Interconversion of Inverse Bicontinuous Cubic Mesophases. Phys. Rev. E 2005, 72, 011502.

(15) Conn, C. E.; Ces, O.; Mulet, X.; Finet, S.; Winter, R.; Seddon, J. M.; Templer, R. H. Dynamics of Structural Transformations between Lamellar and Inverse Bicontinuous Cubic Lyotropic Phases. Phys. Rev. Lett. 2006, 96, 4.

(16) Biggins, J. S.; Warner, M.; Bhattacharya, K. Supersoft Elasticity in Polydomain Nematic Elastomers. Phys. Rev. Lett. 2009, 103, 3.

(17) Pieranski, P. Faceting of Soft Crystals. In Advances in Planar Lipid Bilayers and Liposomes; Iglič, A., Ed.; Elsevier Academic: San Diego, 2011; Vol. 14, pp 1-43.

(18) Rittman, M.; Frischherz, M.; Burgmann, F.; Hartley, P. G.; Squires, A. Direct Visualisation of Lipid Bilayer Cubic Phases Using Atomic Force Microscopy. Soft Matter 2010, 6, 4058.

(19) Munoz, J.; Alfaro, M. C. Rheological and Phase Behaviour of Amphiphilic Lipids. Grasas Aceites (Sevilla, Spain) 2000, 51, 6-.

(20) Plivelic, T. S. ; Labrador, A. L.; Theodor, K.; Gaponov, Y.; Svensson, C.; Nygaard, J.; Cerenius, Y. Proceedings of the 8th Nordic Workshop on Scattering from Soft Matter; Kjeller, Norway, 2011.

(21) Gras, S. L.; Squires, A. M. Dried and Hydrated X-Ray Scattering Analysis of Amyloid Fibrils. In Protein Folding, Misfolding, and Disease; Methods in Molecular Biology, 2011, Vol. 752; pp 147-163.

(22) Ahir, S. V.; Squires, A. M.; Tajbakhsh, A. R.; Terentjev, E. M. Infrared Actuation in Aligned Polymer-Nanotube Composites. Phys. Rev. B 2006, 73, 085420.

(23) De Wolff, P. M. On the Lorentz Factor for Integrated Intensities from Azimuthal and Radial Diffractometer Records of Fiber Patterns. J. Polym. Sci. 1962, 60, S34-.

(24) Hamley, I. W.; Pople, J. A.; Fairclough, J. P. A.; Ryan, A. J.; Booth, C.; Yang, Y. W. Shear-Induced Orientational Transitions in the Body-Centered Cubic Phase of a Diblock Copolymer Gel. Macromolecules 1998, 31, 3906-.

(25) Porcar, L.; Hamilton, W. A.; Butler, P. D.; Warr, G. G. Topological Relaxation of a Shear-Induced Lamellar Phase to Sponge
Equilibrium and the Energetics of Membrane Fusion. Phys. Rev. Lett. 2004, 93, 198301.

(26) Rodríguez-Pérez, J. C.; Hamley, I. W.; Squires, A. M., Infrared Linear Dichroism Spectroscopy on Amyloid Fibrils Aligned by Molecular Combing. Biomacromolecules 2011, 12, 1810.

(27) Rodríguez-Pérez, J. C.; Hamley, I. W.; Gras, S. L.; Squires, A. M. Local Orientational Disorder in Peptide Fibrils Probed by a Combination of Residue-Specific 13C-18O Labelling, Polarised Infrared Spectroscopy and Molecular Combing. Chem. Commun. 2012, 48, 11835.

(28) Briggs, J.; Chung, H.; Caffrey, M. The TemperatureComposition Phase Diagram and Mesophase Structure Characterization of the Monoolein/Water System. J. Phys. II 1996, 6, 723. 